\title{
Statistical Mechanics of Time Domain Ensemble Learning
}

\author{
Seiji MIYOSHI * Tatsuya UEZU ${ }^{\dagger} \quad$ Masato OKADA ${ }^{\ddagger}$
}

June 28, 2018

\section{Abstract}

Conventional ensemble learning combines students in the space domain. On the other hand, in this paper we combine students in the time domain and call it time domain ensemble learning. In this paper, we analyze the generalization performance of time domain ensemble learning in the framework of online learning using a statistical mechanical method. We treat a model in which both the teacher and the student are linear perceptrons with noises. Time domain ensemble learning is twice as effective as conventional space domain ensemble learning.

Keywords: ensemble learning, online learning, generalization error, statistical mechanics

\section{Introduction}

Learning is to infer the underlying rules that dominate data generation using observed data. Observed data are input-output pairs from a teacher and are called examples. Learning can be roughly classified into batch learning and online learning [1. In batch learning, given examples are used more than once. In this paradigm, a student will give the correct answers after training if that student has adequate degree of freedom. However, it is necessary to have a long time and a large memory in which many examples are stored. On the contrary, in online learning examples used once are then discarded. In this case, a student cannot give correct answers for all the examples used in training. However, there are merits, for example, a large memory for storing many examples is not necessary, and it is possible to follow a time variant teacher.

Recently, we 2, 3] analyzed the generalization performance of some models in a framework of online learning using a statistical mechanical method 3, 4, 5. Ensemble learning means to combine many rules or learning machines (called students in this paper) that perform poorly; it has recently attracted the attention of many researchers [6, 7, 8, 9, 2, The diversity or variety of students is important in ensemble learning. We showed that the three well-known rules, Hebbian learning, perceptron learning, and AdaTron learning have different characteristics in their affinities for ensemble learning, that is in "maintaining diversity among students" [3] D In that process, the following points were proven subsidiarily [12, 13, 14 [15]. The student vector doesn't converge in one direction but continues moving in an unlearnable case 10, 11. Therefore, we also analyzed the generalization performance of a student supervised by a moving teacher that goes around a true teacher [4. As a result, it was proven that the generalization error of a student can be smaller than a moving teacher, even if the student only uses examples from the moving teacher. In an actual human society, a teacher observed by a student does not always present the correct answer. In many cases, the teacher is learning and continues to change. Therefore, the analysis of such a model is interesting for considering the analogies between statistical learning theories and an actual human society.

In conventional ensemble learning, the generalization performance is improved by combining students who have diversities. On the other hand, a student does not always converge in one direction but may continue moving in an unlearnable model. Therefore, the generalization performance in such a model must be improved by combining the student itself at different times even if there is only one student 14, 15. Conventional ensemble learning combines students in the space domain. On the other hand, we

\footnotetext{
${ }^{*}$ Department of Electronic Engineering, Kobe City College of Technology, 8-3 Gakuenhigashimachi, Nishi-ku, Kobe-shi, 651-2194 Japan

† Graduate School of Humanities and Sciences, Nara Women's University, Kitauoyahigashi-machi, Nara, 630-8506 Japan

${ }_{\ddagger}^{\ddagger}$ Division of Transdisciplinary Sciences, Graduate School of Frontier Sciences, The University of Tokyo, 5-1-5 Kashiwanoha, Kashiwa-shi, Chiba, 277-8561 Japan

RIKEN Brain Science Institute, 2-1 Hirosawa, Wako-shi, Saitama, 351-0198 Japan

JST PRESTO
} 
introduce a method of combining the students in the time domain; we call this time domain ensemble learning. In this paper, we analyze the generalization performance of the time domain ensemble learning using a statistical mechanical method. We treat a model in which both the teacher and the student are linear perceptrons [2] with noises. We obtain the order parameters and generalization errors analytically in a framework of online learning using a statistical mechanical method.

\section{Model}

In this paper we consider a teacher and a student. They are linear perceptrons with the connection weights $\boldsymbol{B}$ and $\boldsymbol{J}^{m}$, respectively. Here, $m$ denotes the time step. For simplicity, the connection weights of the teacher and the student are simply called the teacher and the student, respectively. Teacher $\boldsymbol{B}=\left(B_{1}, \ldots, B_{N}\right)$, student $\boldsymbol{J}^{m}=\left(J_{1}^{m}, \ldots, J_{N}^{m}\right)$, and input $\boldsymbol{x}^{m}=\left(x_{1}^{m}, \ldots, x_{N}^{m}\right)$ are $N$ dimensional vectors. Each component $B_{i}$ of $\boldsymbol{B}$ is independently drawn from $\mathcal{N}(0,1)$ and fixed, where $\mathcal{N}(0,1)$ denotes a Gaussian distribution with a mean of zero and variance unity. Each component $J_{i}^{0}$ of the initial value $\boldsymbol{J}^{0}$ of $\boldsymbol{J}^{m}$ is independently drawn from $\mathcal{N}(0,1)$. The direction cosine between $\boldsymbol{J}^{m}$ and $\boldsymbol{B}$ is $R^{m}$ and that between $\boldsymbol{J}^{m}$ and $\boldsymbol{J}^{m^{\prime}}$ is $q^{m, m^{\prime}}$. Each component $x_{i}^{m}$ of $\boldsymbol{x}^{m}$ is drawn from $\mathcal{N}(0,1 / N)$ independently. Thus,

$$
\begin{aligned}
\left\langle B_{i}\right\rangle & =0,\left\langle\left(B_{i}\right)^{2}\right\rangle=1, \\
\left\langle J_{i}^{0}\right\rangle & =0,\left\langle\left(J_{i}^{0}\right)^{2}\right\rangle=1, \\
\left\langle x_{i}^{m}\right\rangle & =0,\left\langle\left(x_{i}^{m}\right)^{2}\right\rangle=\frac{1}{N}, \\
R^{m} & \equiv \frac{\boldsymbol{B} \cdot \boldsymbol{J}^{m}}{\|\boldsymbol{B}\|\left\|\boldsymbol{J}^{m}\right\|}, \\
q^{m, m^{\prime}} & \equiv \frac{\boldsymbol{J}^{m} \cdot \boldsymbol{J}^{m^{\prime}}}{\left\|\boldsymbol{J}^{m}\right\|\left\|\boldsymbol{J}^{m^{\prime}}\right\|},
\end{aligned}
$$

where $\langle\cdot\rangle$ denotes a mean.

Figure \illustrates the relationship among teacher $\boldsymbol{B}$, students $\boldsymbol{J}^{m}$ and $\boldsymbol{J}^{m^{\prime}}$ and the direction cosines $R^{m}, R^{m^{\prime}}$, and $q^{m, m^{\prime}}$.

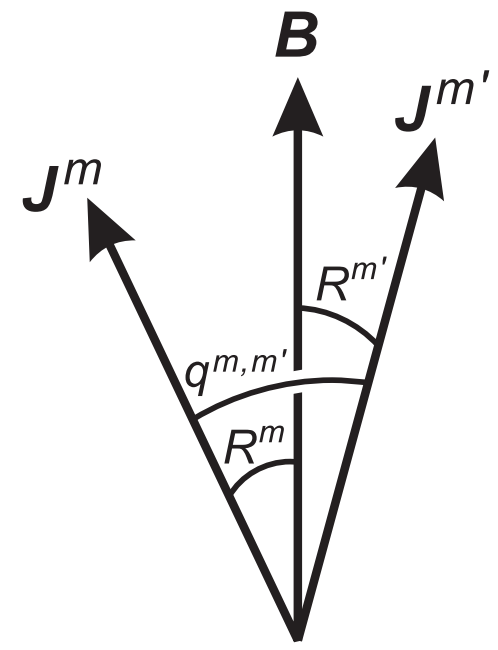

Figure 1: Teacher $\boldsymbol{B}$ and students $\boldsymbol{J}^{m}$ and $\boldsymbol{J}^{m^{\prime}} . R^{m}, R^{m^{\prime}}$, and $q^{m, m^{\prime}}$ are direction cosines.

In this paper, the thermodynamic limit $N \rightarrow \infty$ is also treated. Therefore,

$$
\|\boldsymbol{B}\|=\sqrt{N}, \quad\left\|\boldsymbol{J}^{0}\right\|=\sqrt{N}, \quad\left\|\boldsymbol{x}^{m}\right\|=1 .
$$

Generally, the norm $\left\|\boldsymbol{J}^{m}\right\|$ of the student changes as the time step proceeds. Therefore, the ratios $l^{m}$ of the norm to $\sqrt{N}$ are introduced and are called the length of the student. That is, $\left\|\boldsymbol{J}^{m}\right\|=l^{m} \sqrt{N}$. 
Both the teacher and the student are linear perceptrons. Their outputs are $v^{m}+n_{B}^{m}$ and $u^{m} l^{m}+n_{J}^{m}$, respectively. Here,

$$
\begin{aligned}
v^{m} & =\boldsymbol{B} \cdot \boldsymbol{x}^{m}, \\
u^{m} l^{m} & =\boldsymbol{J}^{m} \cdot \boldsymbol{x}^{m}, \\
n_{B}^{m} & \sim \mathcal{N}\left(0, \sigma_{B}^{2}\right), \\
n_{J}^{m} & \sim \mathcal{N}\left(0, \sigma_{J}^{2}\right),
\end{aligned}
$$

where $\mathcal{N}\left(0, \sigma^{2}\right)$ denotes a Gaussian distribution with a mean of zero and variance $\sigma^{2}$. That is, the outputs of the teacher and the student include independent Gaussian noises with variances of $\sigma_{B}^{2}$ and $\sigma_{J}^{2}$, respectively. Then, $v^{m}$ and $u^{m}$ obey Gaussian distributions with a mean of zero and variance unity.

Let us define the error $\epsilon_{S}^{m}$ between the teacher $\boldsymbol{B}$ and the student $\boldsymbol{J}^{m}$ alone by the squared error of their outputs:

$$
\epsilon_{S}^{m} \equiv \frac{1}{2}\left(v^{m}+n_{B}^{m}-u^{m} l^{m}-n_{J}^{m}\right)^{2} .
$$

Student $\boldsymbol{J}^{m}$ adopts the gradient method as a learning rule and uses input $\boldsymbol{x}$ and an output of teacher $\boldsymbol{B}$ for updates. That is,

$$
\begin{aligned}
\boldsymbol{J}^{m+1} & =\boldsymbol{J}^{m}-\eta \frac{\partial \epsilon_{S}^{m}}{\partial \boldsymbol{J}^{m}} \\
& =\boldsymbol{J}^{m}+\eta\left(v^{m}+n_{B}^{m}-u^{m} l^{m}-n_{J}^{m}\right) \boldsymbol{x}^{m}
\end{aligned}
$$

where $\eta$ denotes the learning rate of the student and is a constant number. Generalizing the learning rule, eq. (13) can be expressed as

$$
\boldsymbol{J}^{m+1}=\boldsymbol{J}^{m}+f^{m} \boldsymbol{x}^{m},
$$

where $f$ denotes a function that represents the update amount and is determined by the learning rule.

\section{Theory}

\subsection{Generalization error}

Ensemble learning means to improve performance by combining many students that perform poorly. On the other hand, we use just one student and combine its copies (hereafter called 'brothers') at different time steps in this paper. Conventional ensemble learning combines students in the space domain; on the other hand, we combine students in the time domain. In this paper $K$ brothers $\boldsymbol{J}^{m_{1}}, \boldsymbol{J}^{m_{2}}, \ldots, \boldsymbol{J}^{m_{K}}$ are combined. Here, $m_{1} \leq m_{2} \leq \ldots \leq m_{K}$. We use the squared error $\epsilon$ for new input $\boldsymbol{x}$. Here, it is assumed that the Gaussian noises of eqs.(9) and (10) are independently added to the teacher and each brother of the ensemble, respectively. The weight of each brother $\boldsymbol{J}^{m_{k}}$ of the ensemble satisfies $C_{k}>0$. That is, the error of the ensemble is

$$
\epsilon=\frac{1}{2}\left(\boldsymbol{B} \cdot \boldsymbol{x}+n_{B}-\sum_{k=1}^{K} C_{k}\left(\boldsymbol{J}^{m_{k}} \cdot \boldsymbol{x}+n_{k}\right)\right)^{2} .
$$

Here, $n_{B} \sim \mathcal{N}\left(0, \sigma_{B}^{2}\right)$ and $n_{k} \sim \mathcal{N}\left(0, \sigma_{J}^{2}\right)$.

A goal of statistical learning theory is to theoretically obtain generalization errors. Since a generalization error is the mean of errors over the distribution of the new input $\boldsymbol{x}$ and noises $n_{B}, n_{k}, k=1, \ldots, K$, the generalization error $\epsilon_{g}$ of the ensemble is calculated as follows:

$$
\begin{aligned}
\epsilon_{g} & =\int d \boldsymbol{x} d n_{B}\left(\prod_{k=1}^{K} d n_{k}\right) p(\boldsymbol{x}) p\left(n_{B}\right)\left(\prod_{k=1}^{K} p\left(n_{k}\right)\right) \epsilon \\
& =\int d v\left(\prod_{k=1}^{K} d u_{k}\right) d n_{B}\left(\prod_{k=1}^{K} d n_{k}\right) p\left(v,\left\{u_{k}\right\}\right) p\left(n_{B}\right)\left(\prod_{k=1}^{K} p\left(n_{k}\right)\right) \frac{1}{2}\left(v+n_{B}-\sum_{k=1}^{K} C_{k}\left(u_{k} l^{m_{k}}+n_{k}\right)\right)^{2} \\
& =\frac{1}{2}\left(1-2 \sum_{k=1}^{K} C_{k} l^{m_{k}} R^{m_{k}}+2 \sum_{k=1}^{K} \sum_{k^{\prime}>k}^{K} C_{k} C_{k^{\prime}} l^{m_{k}} l^{m_{k^{\prime}}} q^{m_{k}, m_{k^{\prime}}}+\sum_{k=1}^{K} C_{k}^{2}\left(l^{m_{k}}\right)^{2}+\sigma_{B}^{2}+\sum_{k=1}^{K} C_{k}^{2} \sigma_{J}^{2}\right),
\end{aligned}
$$

where $v=\boldsymbol{B} \cdot \boldsymbol{x}$ and $u_{k} l^{m_{k}}=\boldsymbol{J}^{m_{k}} \cdot \boldsymbol{x}$. We executed integration using the following: $v$ and $u_{k}$ obey $\mathcal{N}(0,1)$. The covariance between $v$ and $u_{k}$ is $R^{m_{k}}$, that between $u_{k}$ and $u_{k^{\prime}}$ is $q^{m_{k}, m_{k^{\prime}}} \cdot n_{B}$ and $n_{k}$ are independent from other probabilistic variables. 


\subsection{Differential equations for order parameters and their analytical solutions}

In this paper, we examine the thermodynamic limit $N \rightarrow \infty$. Therefore, updates for (13) or (14) must be executed $O(N)$ times for the order parameters $l, R$, and $q$ to change by $O(1)$. Thus, the continuous times $t_{1}, \ldots, t_{K}$, which are the time steps $m_{1}, \ldots, m_{K}$ normalized by the dimension $N$, are introduced as the superscripts that stand for the learning process. To simplify the analysis, we introduced the following auxiliary order parameters:

$$
\begin{aligned}
r^{t} & \equiv l^{t} R^{t}, \\
Q^{t, t^{\prime}} & \equiv l^{t} l^{t^{\prime}} q^{t, t^{\prime}} .
\end{aligned}
$$

The simultaneous differential equations in deterministic forms [16, which describe the dynamical behaviors of order parameters, have been obtained based on the self-averaging of thermodynamic limits as follows:

$$
\begin{aligned}
\frac{d l^{t}}{d t} & =\left\langle f^{t} u^{t}\right\rangle+\frac{\left\langle\left(f^{t}\right)^{2}\right\rangle}{2 l^{t}}, \\
\frac{d r^{t}}{d t} & =\left\langle f^{t} v^{t}\right\rangle, \\
\frac{d Q^{t, t^{\prime}}}{d t^{\prime}} & =l^{t}\left\langle f^{t^{\prime}} \bar{u}^{t}\right\rangle,
\end{aligned}
$$

where $t^{\prime} \geq t$ and $\bar{u}^{t}=\boldsymbol{x}^{t^{\prime}} \cdot \boldsymbol{J}^{t} / l^{t} \sim \mathcal{N}(0,1)$.

Since linear perceptrons are used in this paper, the sample averages that appear in the above equations can be easily calculated as follows:

$$
\begin{aligned}
\left\langle f^{t} u^{t}\right\rangle & =\eta\left(r^{t} / l^{t}-l^{t}\right) \\
\left\langle f^{t} v^{t}\right\rangle & =\eta\left(1-r^{t}\right) \\
\left\langle\left(f^{t}\right)^{2}\right\rangle & =\eta^{2}\left(1+\sigma_{B}^{2}+\sigma_{J}^{2}+\left(l^{t}\right)^{2}-2 r^{t}\right) \\
\left\langle f^{t^{\prime}} \bar{u}^{t}\right\rangle & =\eta\left(r^{t}-Q^{t, t^{\prime}}\right) / l^{t}
\end{aligned}
$$

Since all components of teacher $\boldsymbol{B}$ and the initial student $\boldsymbol{J}^{0}$ are independently drawn from $\mathcal{N}(0,1)$ and because the thermodynamic limit $N \rightarrow \infty$ is also used, they are orthogonal to each other in the initial state. That is,

$$
R^{0}=0 .
$$

In addition,

$$
l^{0}=1
$$

and

$$
Q^{t, t}=\left(l^{t}\right)^{2}
$$

using eqs.(5) and (20). Using these initial conditions, we can analytically solve the simultaneous differential equations (21)-27) as follows:

$$
\begin{aligned}
r^{t} & =1-e^{-\eta t}, \\
\left(l^{t}\right)^{2} & =1+\frac{\eta}{2-\eta}\left(\sigma_{B}^{2}+\sigma_{J}^{2}\right)-2 e^{-\eta t}+\left(2-\frac{\eta}{2-\eta}\left(\sigma_{B}^{2}+\sigma_{J}^{2}\right)\right) e^{\eta(\eta-2) t}, \\
Q^{t, t^{\prime}} & =1-e^{-\eta t}+e^{-\eta t^{\prime}}+\left(\left(l^{t}\right)^{2}-1\right) e^{-\eta\left(t^{\prime}-t\right)} .
\end{aligned}
$$

Substituting eqs.(31)-(33) into eq.(18), the generalization error $\epsilon_{g}$ can be analytically obtained as a function of time $t_{k}, k=1, \ldots, K$ as follows:

$$
\begin{aligned}
\epsilon_{g} & =\frac{1}{2}\left[1-2 \sum_{k=1}^{K} C_{k}\left(1-e^{-\eta t_{k}}\right)\right. \\
& +2 \sum_{k=1}^{K} \sum_{k^{\prime}>k}^{K} C_{k} C_{k^{\prime}}\left(1-e^{-\eta t_{k}}+e^{-\eta t_{k^{\prime}}}+\left(\bar{\sigma}^{2}-2 e^{-\eta t_{k}}+\left(2-\bar{\sigma}^{2}\right) e^{\eta(\eta-2) t_{k}}\right) e^{-\eta\left(t_{k^{\prime}}-t_{k}\right)}\right) \\
& \left.+\sum_{k=1}^{K} C_{k}^{2}\left(1+\bar{\sigma}^{2}-2 e^{-\eta t_{k}}+\left(2-\bar{\sigma}^{2}\right) e^{\eta(\eta-2) t_{k}}\right)+\sigma_{B}^{2}+\sum_{k=1}^{K} C_{k}^{2} \sigma_{J}^{2}\right] \\
\bar{\sigma}^{2} & =\frac{\eta}{2-\eta}\left(\sigma_{B}^{2}+\sigma_{J}^{2}\right)
\end{aligned}
$$




\section{Results and Discussion}

The dynamical behaviors of $l^{t}$ and $R^{t}$ have been analytically obtained using eqs. (19), (31), and (32). Figures 2 and 3 show some examples of the analytical results and the corresponding simulation results, where $N=2000$. In these figures, the curves represent theoretical results. The symbols represent simulation results. Figure 2 shows the results of $\sigma_{B}^{2}=\sigma_{J}^{2}=0.0$ and no noise. Figure [3] shows the result of $\sigma_{B}^{2}=\sigma_{J}^{2}=0.2$.

Focusing on the signs of the powers of the exponential functions in eq.(32), we can see that $l^{t}$ diverges if the learning rate satisfies $0>\eta$ or $\eta>2 . l^{t}$ converges to

$$
l^{\infty}=\sqrt{1+\bar{\sigma}^{2}}
$$

if $0<\eta<2$. Equations (19) and (31) show that $R^{t}$ converges to

$$
R^{\infty}=1 / l^{\infty} .
$$

Therefore, we can see that $l^{\infty}=R^{\infty}=1$ in the case of no noise and $l^{\infty}>1, R^{\infty}<1$ in the case of noise.

Since eq.(32) shows

$$
\left.\frac{d\left(l^{t}\right)^{2}}{d t}\right|_{t=0} \begin{cases}<0 & \text { when } \eta<\frac{2}{2+\sigma_{B}^{2}+\sigma_{J}^{2}}, \\ =0 & \text { when } \eta=\frac{2}{2+\sigma_{B}^{2}+\sigma_{J}^{2}}, \\ >0 & \text { when } \eta>\frac{2}{2+\sigma_{B}^{2}+\sigma_{J}^{2}}\end{cases}
$$

an equation regarding $t$

$$
\frac{d\left(l^{t}\right)^{2}}{d t}=0
$$

has only one solution

$$
t=\frac{1}{\eta(1-\eta)} \ln \left(2-\frac{\eta}{2}\left(2+\sigma_{B}^{2}+\sigma_{J}^{2}\right)\right),
$$

if the learning rate satisfies $0<\eta<4 /\left(2+\sigma_{B}^{2}+\sigma_{J}^{2}\right)$ and $\eta \neq 1$. Therefore, $l^{t}$ asymptotically approaches unity after becoming larger than unity if $0<\eta<1$ and $l^{t}$ asymptotically approaches unity after becoming smaller than unity if $1<\eta<2$ as shown in Figure 2(a).

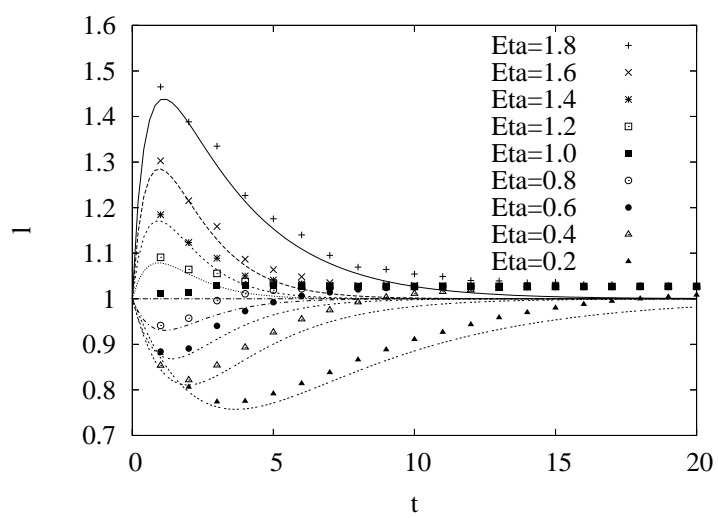

(a) Dynamical behaviors of $l^{t}$.

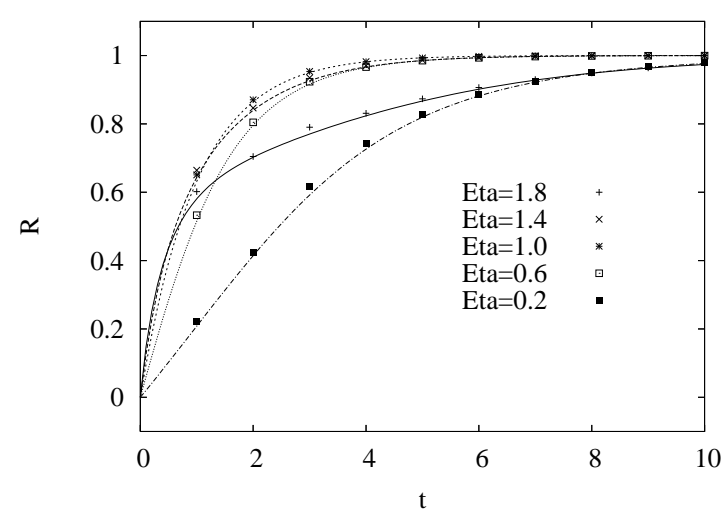

(b) Dynamical behaviors of $R^{t}$.

Figure 2: Dynamical behaviors of $l^{t}$ and $R^{t}$. Theory and computer simulations. $\sigma_{B}^{2}=\sigma_{J}^{2}=0.0 \mathrm{D}$

Equations (19), (31), and (32) show

$$
\left.\frac{d R^{t}}{d t}\right|_{t=0}=\eta .
$$

Therefore, the larger $\eta$ is, the faster $R$ rises as shown in Figs. 2(b) and 3(b). However, eqs.(19), (31), (32), (36), and (37) show

$$
\begin{aligned}
R^{\infty}-R^{t} & =\frac{1}{l^{\infty}}-\frac{r^{t}}{l^{t}} \\
& \rightarrow\left(1+\bar{\sigma}^{2}\right)\left(\bar{\sigma}^{2} e^{-\eta t}+\frac{2-\bar{\sigma}^{2}}{2} e^{\eta(\eta-2) t}\right)
\end{aligned}
$$




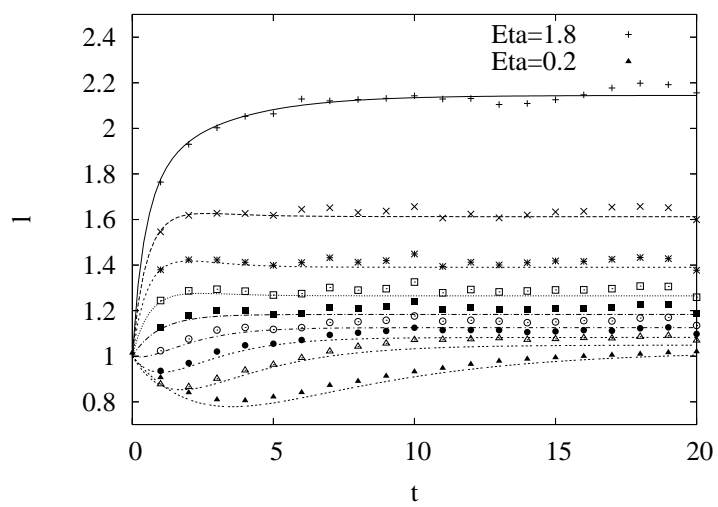

(a) Dynamical behaviors of $l^{t}$.

$\eta=1.8,1.6,1.4,1.2,1.0,0.8,0.6,0.4$ and 0.2 from the top.

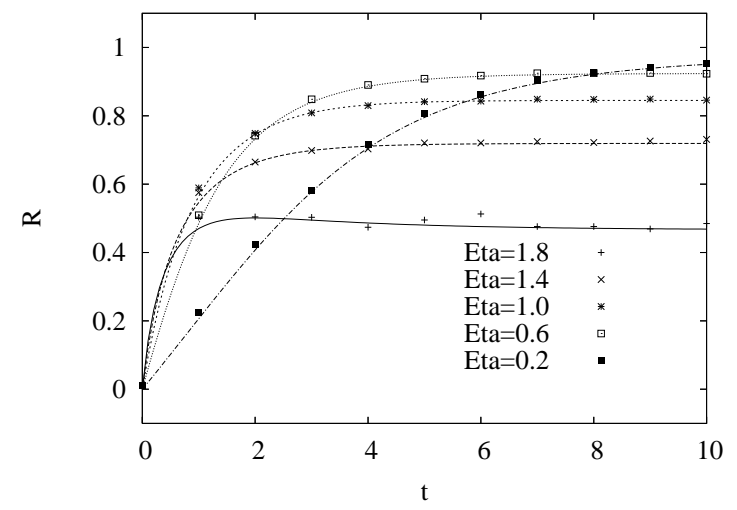

(b) Dynamical behaviors of $R^{t}$.

Figure 3: Dynamical behaviors of $l^{t}$ and $R^{t}$. Theory and computer simulations. $\sigma_{B}^{2}=\sigma_{J}^{2}=0.0 \mathrm{D}$

when $t$ is large. Since eq. (43) is $O\left(e^{-\eta t}\right)$ if $0<\eta \leq 1$ and $O\left(e^{\eta(\eta-2) t}\right)=O\left(e^{\left((\eta-1)^{2}-1\right) t}\right)$ if $1<\eta<2$, the convergence of $R^{t}$ is the fastest when the learning rate satisfies $\eta=1$. This can be confirmed in Figure 2(b) and Figure 3(b). This phenomenon can be understood by the fact that $\eta=1$ is a special condition where the student uses up the information obtained from input $\boldsymbol{x}[5]$.

We analytically obtained the dynamical behaviors of the generalization error $\epsilon_{g}$ and the direction cosine $q$ using eqs.(20) and (32)-(35). Figures 4 and 5 show some examples of the analytical results and the corresponding simulation results, where $N=2000$. In these figures, the curves represent theoretical results. The symbols represent simulation results. $\epsilon_{g}$ has been calculated for the simplest case, that is $K=2, C_{1}=C_{2}=1 / 2$. Other conditions are $\eta=1.0$ and $\sigma_{B}^{2}=\sigma_{J}^{2}=0.2$. In the computer simulations, $\epsilon_{g}$ was obtained by averaging the squared errors for $10^{4}$ random inputs at each time step.

Figure 4 shows the relationship between $t_{2}-t_{1}$ and $\epsilon_{g}, q^{t_{1}, t_{2}}$ in the case of a constant $t_{1}$. When $t_{2}-t_{1}$ increases, $\epsilon_{g}$ increases monotonically, remains constant, or decreases monotonically depending on the values of $\eta$. We prove this in the following. Equation (34) shows that $\epsilon_{g(K=1)}$ is

$$
\epsilon_{g(K=1)}=\frac{1}{2}\left(\frac{\eta}{2-\eta}\left(\sigma_{B}^{2}+\sigma_{J}^{2}\right)+\left(2-\frac{\eta}{2-\eta}\left(\sigma_{B}^{2}+\sigma_{J}^{2}\right)\right) e^{\eta(\eta-2) t}\right) .
$$

Therefore, $\epsilon_{g(K=1)}$ decreases monotonically, remains constant, or increases monotonically as time $t$ proceeds. The necessary and sufficient conditions for the above three phenomena are

$$
\begin{aligned}
\eta & <\frac{4}{2+\sigma_{B}^{2}+\sigma_{J}^{2}}, \\
\eta & =\frac{4}{2+\sigma_{B}^{2}+\sigma_{J}^{2}}, \\
\eta & >\frac{4}{2+\sigma_{B}^{2}+\sigma_{J}^{2}},
\end{aligned}
$$

respectively. Since the output of the ensemble is the weighted sum of the outputs of the brothers, the generalization error for $K>1$ also decreases monotonically, remains constant, or increases monotonically. The necessary and sufficient conditions for these three phenomena are also shown in eqs. (45)-(47). Since the condition of Fig. 4(a) agrees with eq.(45), the generalization error decreases monotonically. Equations (20), (32), (33), and (36) show that $q^{t_{1}, t_{2}}$ in the case of $t_{1}=0$ asymptotically approaches zero when $t_{2}-t_{1} \rightarrow \infty$ as shown in Fig. 4(b). This means that after a long time the student is orthogonal with its initial condition.

Since the order parameters and $\epsilon_{g}$ have been explicitly obtained as functions of $t$ and $t^{\prime}$ as eqs. 31(34) in this paper, the relationships between $t_{1}$ and $\epsilon_{g}, q^{t, t^{\prime}}$ in the case of constant time interval of the brothers or constant $t_{k+1}-t_{k}$ can be calculated. Figure 5 shows the relationship between $t_{1}$ and $\epsilon_{g}$, $q^{t_{1}, t_{2}}$ in the case of constant $t_{2}-t_{1}$. For the same reason as in Fig. 4l (a), the generalization error $\epsilon_{g}$ also decreases monotonically in Fig. [5(a). Figure 5](b) shows that $q^{t_{1}, t_{2}}$ converges to a smaller value than unity in the case of $t_{2}-t_{1} \neq 0.0$. This means that the student itself continues to move after the order parameters $l, R$, and $q$ reach a steady state.

In Figs. 4 and 5 the generalization error $\epsilon_{g}$ and the direction cosine $q^{t_{1}, t_{2}}$ seem to reach a almost steady state by $t_{2}-t_{1}>5$ or $t_{1}>5$. The behaviors of $\epsilon_{g}$ when the leading time $t_{1} \rightarrow \infty$ or the 


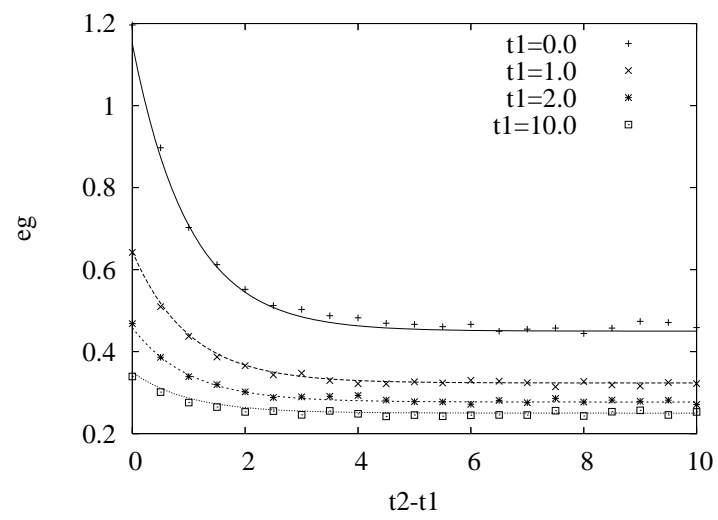

(a) $\epsilon_{g}$

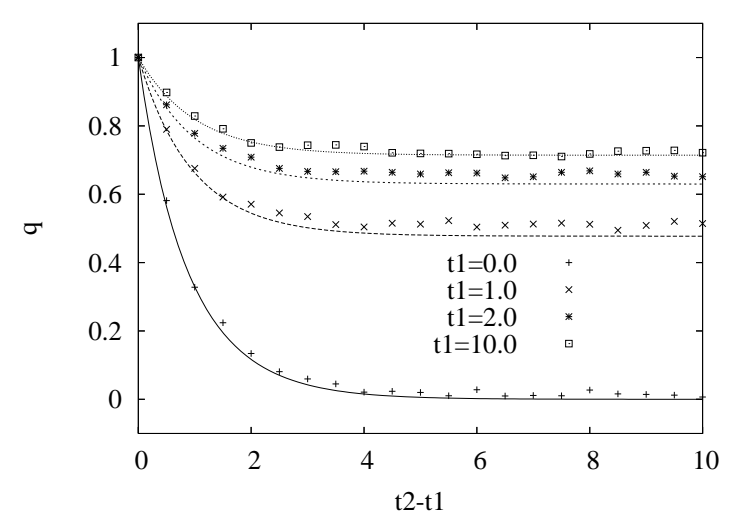

(b) $q^{t_{1}, t_{2}}$

Figure 4: Relationship between $t_{2}-t_{1}$ and $\epsilon_{g}, q^{t_{1}, t_{2}}$ in the case of constant leading time $t_{1}$. Theory and computer simulations. $\eta=1.0, \sigma_{B}^{2}=\sigma_{J}^{2}=0.2 \mathrm{D}$

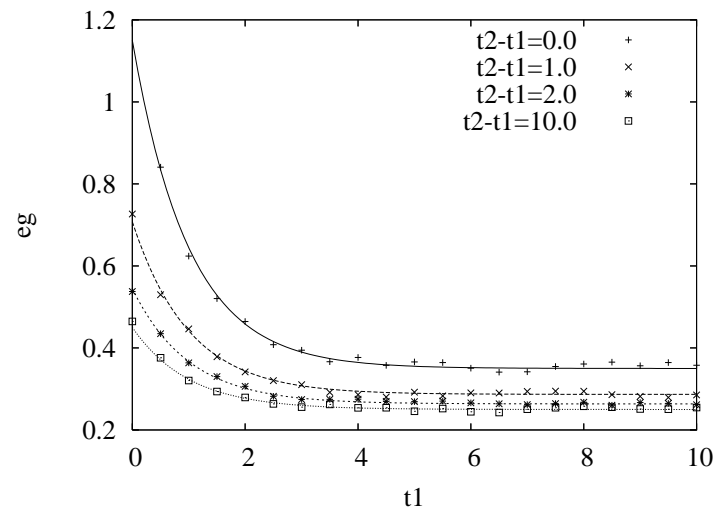

(a) $\epsilon_{g}$

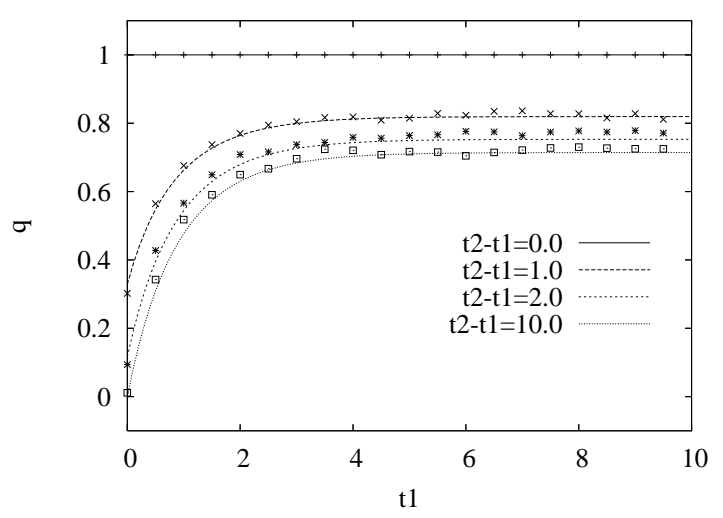

(b) $q^{t_{1}, t_{2}}$

Figure 5: Relationship between $t_{1}$ and $\epsilon_{g}, q^{t_{1}, t_{2}}$ in the case of constant time interval $t_{2}-t_{1}$. Theory and computer simulations. $\eta=1.0, \sigma_{B}^{2}=\sigma_{J}^{2}=0.2 \mathrm{D}$ 
time interval $t_{k+1}-t_{k} \rightarrow \infty$ can be theoretically obtained since the generalization error and the order parameters have been analytically obtained as functions of $t_{k}, k=1, \ldots, K$, as shown in eq. (34).

At first, eqs. (32) and (34) show that $\left(l^{t}\right)^{2}$ diverges unless $0<\eta<2$. Therefore, the generalization error diverges unless $0<\eta<2$. If $0<\eta<2$, the generalization error can be discussed as follows:

When $t_{1} \rightarrow \infty$, from eqs. (34) and (35), we obtain

$$
\begin{aligned}
\epsilon_{g} & =\frac{1}{2}\left[1-2 \sum_{k=1}^{K} C_{k}+2 \sum_{k=1}^{K} \sum_{k^{\prime}>k}^{K} C_{k} C_{k^{\prime}}\left(1+\frac{\eta}{2-\eta}\left(\sigma_{B}^{2}+\sigma_{J}^{2}\right) e^{-\eta\left(t_{k^{\prime}}-t_{k}\right)}\right)\right. \\
& \left.+\sum_{k=1}^{K} C_{k}^{2}\left(1+\frac{\eta}{2-\eta}\left(\sigma_{B}^{2}+\sigma_{J}^{2}\right)\right)+\sigma_{B}^{2}+\sum_{k=1}^{K} C_{k}^{2} \sigma_{J}^{2}\right] .
\end{aligned}
$$

In addition, when the time interval $t_{k+1}-t_{k} \rightarrow \infty$, from eq. (48), we obtain

$$
\epsilon_{g}=\frac{1}{2}\left[1-2 \sum_{k=1}^{K} C_{k}+2 \sum_{k=1}^{K} \sum_{k^{\prime}>k}^{K} C_{k} C_{k^{\prime}}+\sum_{k=1}^{K} C_{k}^{2}\left(1+\frac{\eta}{2-\eta}\left(\sigma_{B}^{2}+\sigma_{J}^{2}\right)\right)+\sigma_{B}^{2}+\sum_{k=1}^{K} C_{k}^{2} \sigma_{J}^{2}\right] .
$$

Equation (49) shows that the generalization error decreases as the learning rate $\eta$ decreases regardless of $K$ when $t_{1} \rightarrow \infty$ and $t_{k+1}-t_{k} \rightarrow \infty$.

In addition, when the weights are uniform or $C_{k}=C=1 / K$, from eq.(49), we obtain

$$
\epsilon_{g}=\frac{1}{2 K}\left(\frac{\eta}{2-\eta}\left(\sigma_{B}^{2}+\sigma_{J}^{2}\right)\right)+\frac{1}{2}\left(\sigma_{B}^{2}+\frac{1}{K} \sigma_{J}^{2}\right) .
$$

Here, considering the special case $K=1$, we obtain

$$
\epsilon_{g}=\frac{1}{2}\left(\frac{\eta}{2-\eta}\left(\sigma_{B}^{2}+\sigma_{J}^{2}\right)\right)+\frac{1}{2}\left(\sigma_{B}^{2}+\sigma_{J}^{2}\right) .
$$

If $\boldsymbol{B}=\boldsymbol{J}^{t_{1}}$ is true, the generalization error must equal the residual error

$$
\epsilon_{g}=\frac{1}{2}\left(\sigma_{B}^{2}+\sigma_{J}^{2}\right)
$$

caused by noise from eq.(15), which is the definition of error. Therefore, the difference between eq. (51) and eq. (152)

$$
\frac{1}{2}\left(\frac{\eta}{2-\eta}\left(\sigma_{B}^{2}+\sigma_{J}^{2}\right)\right)
$$

is caused by the disagreement between $\boldsymbol{B}$ and $\boldsymbol{J}^{t_{1}}$.

Next, let us consider another special case, $K=\infty$. If and only if

$$
\boldsymbol{B}=\lim _{K \rightarrow \infty} \frac{1}{K} \sum_{k=1}^{K} \boldsymbol{J}^{t_{k}}
$$

the generalization error must equal the residual error

$$
\epsilon_{g}=\frac{1}{2} \sigma_{B}^{2}
$$

caused by noise from eq. (15), which is the definition of error. Equation (54) is true since eq.(50) equals eq.(55) when $K=\infty$.

In addition, if $\sigma_{B}^{2}=\sigma_{J}^{2}=\sigma^{2}$, eq. (150) changes as follows:

$$
\epsilon_{g}=\left(\frac{1}{2 K} \frac{2+\eta}{2-\eta}+\frac{1}{2}\right) \sigma^{2} .
$$

The relationship between the learning rate $\eta$ and the generalization error $\epsilon_{g}$ can be analytically obtained using eq. (566) when both the leading time $t_{1}$ and the time interval $t_{k+1}-t_{k}$ are large enough, and the uniform weight $C_{k}=C=1 / K$ and $\sigma_{B}^{2}=\sigma_{J}^{2}=0.5$. Figure [6] shows the analytical results and the corresponding simulation results. In the computer simulations, $N=2000$, the leading time $t_{1}=10$, and the time interval $t_{k+1}-t_{k}=10\left(t_{1}=t_{k+1}-t_{k}=20\right.$ when $\left.\eta=0.2\right)$, we obtained $\epsilon_{g}$ by averaging the squared errors for $10^{4}$ random inputs at each time step. Figure 6 confirms the following. The generalization error decreases as the learning rate $\eta$ decreases. The generalization error decreases and converges to the residual error $\frac{1}{2} \sigma_{B}^{2}$ as $K$ increases. 


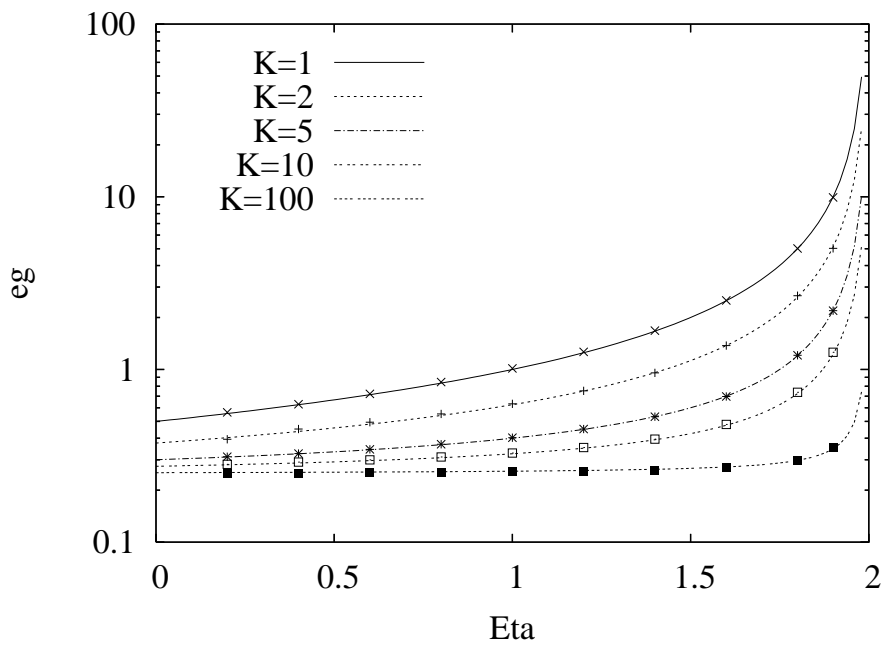

Figure 6: Relationship between the learning rate $\eta$ and the generalization error $\epsilon_{g}$, when both the leading time $t_{1}$ and the time interval $t_{k+1}-t_{k}$ are large enough. Theory and computer simulations. Conditions are $C_{k}=C=1 / K$ and $\sigma_{B}^{2}=\sigma_{J}^{2}=0.5$.

In addition, if the learning rate satisfies $\eta=1$, eq. (56) becomes

$$
\epsilon_{g}=\left(\frac{3}{2 K}+\frac{1}{2}\right) \sigma^{2} .
$$

Equation (57) refers to the generalization error $\epsilon_{g}$ of $K=\infty$, which is $1 / 4$ times of that of $K=1$ when the learning rate satisfies $\eta=1$, the uniform weights $C_{k}=1 / K, \sigma_{B}^{2}=\sigma_{J}^{2}, t_{1} \rightarrow \infty$, and $t_{k^{\prime}}-t_{k} \rightarrow \infty$. Since the generalizaion error $\epsilon_{g}$ of the conventional space domain ensemble learning with $K=\infty, \eta=1$, $C_{k}=1 / K$ and $\sigma_{B}^{2}=\sigma_{J}^{2}$ is $1 / 2$ times of that of $K=1[2$, we can say that the time domain ensemble learning is twice as effective as the conventional space domain ensemble learning. We can explain this difference as follows: In conventional space domain ensemble learning, the similarities among students become high since all students use the same examples for learning. On the other hand, in time domain ensemble learning, the similarities among brothers become low since all brothers use almost totally different examples for learning.

\section{Conclusion}

We analyzed the generalization performance of the time domain ensemble learning in the framework of online learning using a statistical mechanical method. We treated a model in which both the teacher and the student were linear perceptrons with noises. We showed that the time domain ensemble learning is twice as effective as the conventional space domain ensemble learning.

\section{Acknowledgments}

This research was partially supported by the Ministry of Education, Culture, Sports, Science, and Technology of Japan, with Grants-in-Aid for Scientific Research 14084212, 15500151 and 16500093.

\section{References}

[1] D. Saad, (ed.): On-line Learning in Neural Networks (Cambridge University Press, Cambridge, 1998).

[2] K. Hara and M. Okada: J. Phys. Soc. Jpn. 74 (2005) 2966.

[3] S. Miyoshi, K. Hara and M. Okada: Phys. Rev. E 71 (2005) 036116.

[4] S. Miyoshi and M. Okada: J. Phys. Soc. Jpn. 75 (2006) 024003.

[5] S. Miyoshi and M. Okada: J. Phys. Soc. Jpn. 75 (2006) 044002. 
[6] Y. Freund, and R. E. Schapire: Journal of Japanese Society for Artificial Intelligence, 14 (1999) 771 [in Japanese, translation by N. Abe].

[7] http://www.boosting.org/

[8] A. Krogh, and P. Sollich: Phys. Rev. E 55 (1997) 811.

[9] R. Urbanczik: Phys. Rev. E 62 (2000) 1448.

[10] J. I. Inoue and H. Nishimori: Phys. Rev. E 55 (1997) 4544.

[11] J. I. Inoue, H. Nishimori and Y. Kabashima: cond-mat/9708096 (1997).

[12] S. Miyoshi, K. Hara and M. Okada: Proc. The Seventh Workshop on Information-Based Induction Sciences (2004) 178 [in Japanese].

[13] S. Miyoshi, K. Hara and M. Okada: IEICE Technical Report, NC2004-214 (2005) 123 [in Japanese].

[14] Y. Maeda: Master thesis, Graduate School of Humanities and Sciences, Nara Women's University, (2002)

[15] T. Uezu, Y. Maeda and S. Yamaguchi: (2006) [in preparation].

[16] H. Nishimori: Statistical Physics of Spin Glasses and Information Processing: An Introduction (Oxford University Press, Oxford, 2001). 